Methotrexate on Circulating Biomarkers of Synovium, Cartilage and Bone Metabolism: Potential Utility for Clinical Development Decision Making in Rheumatoid Arthriti [abstract]. Arthritis Rheumatol. 2016; 68 (suppl 10).

Disclosure of Interest: C. Kjelgaard-Petersen: None declared, T. Christensen: None declared, P. Hägglund: None declared, M. Karsdal Shareholder of: Nordic Bioscience A/S, Employee of: Nordic Bioscience A/S, C. Thudium Employee of: Nordic Bioscience A/S, A.-C. Bay-Jensen Shareholder of: Nordic Bioscience A/S, Employee of: Nordic Bioscience A/S

DOI: 10.1136/annrheumdis-2017-eular.6788

\section{AB0063 THE PROTECTIVE EFFECT OF PFT $\alpha$ ON ALCOHOL-INDUCED OSTEONECROSIS OF THE FEMORAL HEAD}

C. Yixuan. Orthopedics, Shanghai Jiao Tong University Affiliated Sixth People's Hospital, Shanghai, China

Background: Epidemiologic studies have shown that alcohol plays a pivotal role in the development of osteonecrosis of the femoral head (ONFH). However, few studies have discussed the pathogenesis of or interventions for alcohol-induced $\mathrm{ONFH}$.

Objectives: The aim of this study was to explore the underlying mechanism of alcohol-induced ONFH and the protective effect of pifithrin- $\alpha$ (PFT $\alpha$ ).

Results: Through a series of in vitro assessments, we found that ethanol treatment significantly activated $\mathrm{p} 53$, suppressed $\mathrm{Wnt} / \beta$-catenin signaling and inhibited osteogenic-related proteins, including runt-related transcription factor 2 (RUNX2), osteocalcin (OCN), osteopontin (OPN) and collagen I (COL1). Furthermore, by separating the cytoplasmic and nuclear proteins, we found that ethanol inhibited osteogenesisby impairing the accumulation of $\beta$-cateninin both the cytoplasm and nucleus in human bone mesenchymal stem cells (hBMSCs), which resulted from activating glycogen synthase kinase-3 $\beta$ (GSK$3 \beta)$. In this in vivo study, we established alcohol-induced ONFH in rats and investigated the protective effect of PFT $\alpha$. Micro-CT, hematoxylin \& eosin (H\&E) staining, immunohistochemical analyses, immunofluorescence staining, TUNEL staining, and fluorochrome labeling were performed to reveal the PFT $\alpha$-induced therapeutic effects. H\&E findings combined with TUNEL, caspase-3-cleaved immunohistochemical staining, and micro-CT images revealed obvious ONFH in the alcohol-administered rats, whereas significantly less osteonecrosis developed in the rats injected with PFT $\alpha$. As the initiator of osteogenesis, RUNX2 and its downstream targets $\mathrm{OCN}, \mathrm{OPN}, \mathrm{COL} 1$ were immunostained in the femoral heads. These results indicated that those osteogenic-related proteins were significantly decreased in the alcohol-administered rats, whereas these results were reversed in the PFT $\alpha$-injected rats. Fluorochrome labeling showed a similar result in that alcohol significantly reduced the osteogenic activity in the rat femoral head, which was blocked by the injection of PFT $\alpha$.

Conclusions: Pifithrin- $\alpha$, a p53 inhibitor, was able to block the ethanol-triggered activation of p53 in hBMSCs and alcohol-induced ONFH in a rat model. Its antagonistic effect against ethanol's effect on hBMSCs could be a clinical strategy to prevent the development of alcohol-induced ONFH.

Acknowledgements: The current study was supported by grants from the National Natural Science Foundation of China (no. 81272003 andno. 81301572) and the SMC-Chen Xing Plan for Splendid Young Investigators of Shanghai Jiao Tong University.

Disclosure of Interest: None declared

DOI: 10.1136/annrheumdis-2017-eular.1981

\section{AB0064 ADIPOSE STROMAL CELLS EXERT SPECIFIC EFFECTS ON OSTEOARTHRITIC SYNOVIAL MACROPHAGES}

C. Manferdini ${ }^{1}$, F. Paolella ${ }^{1}$, E. Gabusi ${ }^{1}$, L. Gambari ${ }^{2}$, A. Piacentini ${ }^{1}$ S. Fleury-Cappellesso ${ }^{3}$, A. Barbero ${ }^{4}$, M. Murphy $^{5}$, G. Lisignoli ${ }^{1} .{ }^{1}$ Laboratory of Immunorheumatology and tissue regeneration; ${ }^{2}$ RAMSES Laboratory, Rizzoli Orthopaedic Institute, Bologna, Italy; ${ }^{3}$ 4EFS-Pyrénéés-Méditerranéé, Toulouse, France; ${ }^{4}$ Department of Biomedicine, University Hospital Basel, Basel, Switzerland; ${ }^{5}$ Regenerative Medicine Institute, Galway, Ireland

Background: Osteoarthritis $(\mathrm{OA})$ is the most common joint disease and the major cause of pain and disability in the aging population [1]. Adipose stromal cells (ASC) are promising candidate for cell therapy in OA, because they have immunomodulatory, trophic and differentiation capacities [2,3]. Synovial inflammation is accepted as important OA feature for the symptoms and disease progression [4]. Synovial tissue is mainly composed of synovial fibroblasts (SF), macrophages (SM) and a low percentage of other cell types [5].

Objectives: Aim of the study was to analyze the effects of adipose stromal cells in co-culture with SF and SM.

Methods: GMP clinical grade ASC were isolated from subcutaneous adipose tissue. Synoviocytes were isolated from synovial tissue of OA patients undergoing total joint replacement. Synovial cells at passage 1 and 5 were analyzed for: 1 . different phenotypical markers by flow cytometric analysis, 2 . inflammatory factors by multiplex immunoassay, 3.anabolic and degradative factors by qRT-PCR. Both p.1 (mix of SF and SM) and p.5 (only SF) synovial cells, as different cell models, were co-cultured with adipose stem cells (ASC) to define their effects. Furthermore macrophages type 1 (M1) were isolated and co-cultured with ASC. Results: Synoviocytes at passage 1 were positive to typical markers of SM
(CD14,CD16,CD68, CD80,CD163) and SF (CD55,CD73,CD90,CD105,CD106), whereas at passage 5 were positive only to SF markers and showed a higher percentage of CD55 and CD106. At p.1 synovial cells released a significantly higher amount of all inflammatory (IL6,CXCL8,CCL2,CCL3,CCL5) and anabolic (IL10) factors than those at p.5. Moreover, p.1 synovial cells expressed also higher amount of some degradative factors (MMP13, S100A8, S100A9) than p.5 synovial cells. Co-culture experiments showed that the amount of SM in p.1 synovial cells specifically orchestrate the up or down-modulation of some inflammatory (IL6,CXCL8,CCL2,CCL3,CCL5) and degradative factors (ADAMTS5,MMP13, S100A8,S100A9) analyzed. Interestingly, p.5 synovial cells induced all factors analyzed, except CCL5. Finally, we demonstrated that ASC effects were strictly dependent by $\mathrm{M} 1$, that decreased the release of typical macrophages cytokines (IL1 $\beta$, IL6, TNF $\alpha$ and CCL3/MIP1 $\alpha$ ) and that ASC effects are responsible for the switching by M1 like inflammatory macrophages to M2 like phenotype mainly due to PGE2 involvement.

Conclusions: These data demonstrate that the GMP-ASC effects on OA synovial inflammation are strictly dependent by macrophages, that orchestrate the switching activated-M1 inflammatory macrophages to a M2-like phenotype, mainly through PGE2

References:

[1] Loeser RF (2012) Arthritis Rheum 64:1697-707.

[2] C. Manferdini (2013) Arthritis Rheum 65:1271-81.

[3] M. Maumus (2013) Stem Cell Research 11, 834-844.

[4] Y. Henrotin (2014) Semin Arthritis Rheum 43(5):579-87.

[5] MD. Smith (2011) The Open Rheumatology Journal 5:100-6.

Acknowledgements: This research was supported by Horizon 2020 Programme (project ADIPOA2, grant agreement no: 643809). The materials presented and views expressed here are the responsibility of the authors only. The EU Commission takes no responsibility for any use made of the information set out.

Disclosure of Interest: None declared

DOI: 10.1136/annrheumdis-2017-eular.6081

\section{AB0065 2-CARBA-CYCLIC PHOSPHATIDIC ACID SUPPRESSES EXPRESSION OF CARTILAGE DEGRADING ENZYMES SUCH AS MMP-13 IN INFLAMMATORY SYNOVIAL FIBROBLASTS AND ARTICULAR CHONDROCYTES INDUCED BY IL-1 BETA AND/OR TNF ALFA}

I. Masuda ${ }^{1,2}$, K. Okada ${ }^{3}$, H. Yamanaka ${ }^{1}$, S. Momohara ${ }^{1,4}{ }^{1}{ }^{1}$ Inst. of Rheumatology, Tokyo Women's Medical University, Tokyo; ${ }^{2}$ Rheumatology, Inst. for Rheumatic diseases, Jyujyo-Takeda Rehabilitation Hospital, Kyoto; ${ }^{3}$ SANSHO, CO., Ltd; ${ }^{4}$ Keio University, Tokyo, Japan

Background: Cyclic phosphatidic acid (cPA) is one of bioactive lipid, has been implicated as a mediator of various biological effects including inhibitory effects of proliferation, invasion and metastasis of cancer cells. CPA is naturally occurring mediator even exists in human serum. 2-carba-cPA (2ccPA) is structurally modified formula of CPA and has shown improved bioactivity. We have previously confirmed that 2ccPA stimulated HAS-2 production on human osteoarthritic chondrocytes and synovial fibroblasts (SFs) in vitro. Furthermore, intra-articular administration of 2ccPA has shown its suppressing effect of pain, swelling, and articular cartilage destruction in rabbit experimental osteoarthritis (OA). We have shown that 2ccPA might had direct inhibitory effect of cartilage degrading enzymes on rheumatoid synovial fibroblasts (SFs) in vitro. Inflammatory arthritis such as rheumatoid arthritis (RA) and early stage of OA involves synovial inflammation and subsequent production of cartilage degrading enzymes also from chondrocytes. 2ccPA may be possible another therapeutic option for degenerative arthritis.

Objectives: The aim of this study was to evaluate the direct effects of 2ccPA on cartilage matrix degrading enzymes using SFs and articular chondrocytes under influence of inflammatory cytokines.

Methods: In vitro studies were performed using SFs and chondrocytes obtained from arthritis patients (RA and OA) at joint replacement surgery. First, 2ccPA $0-25 \mu \mathrm{M}$ was added to cell cultures and effects of 2ccPA on ADAMTS-4, -5 , MMP-3, 9, -13 expression was assessed by real time PCR using specific primers to corresponding genes. Beta-actin was used as endogenous expression control for PCR. As we confirmed that 2ccPA had dose-dependent inhibitory effects on expression of above enzymes, the second experiment was performed. SFs or chondrocytes were pre-cultured with IL-1 $\beta(1 \mathrm{ng} / \mathrm{ml})$ and/or TNF- $\alpha(10 \mathrm{ng} / \mathrm{ml})$ for 24 hours, then added $10 \mu \mathrm{M} 2 \mathrm{ccPA}$ to study attenuated effect of 2ccPA on synthesis of above cartilage matrix degrading enzymes. Newly synthesized MMP-3, -13 from SFs or chondrocytes in cultured media after 24 hours of 2ccPA addition were measured by sandwich ELISA.

Results: 2cCPA itself repressed cartilage degrading enzymes, ADAMTS-4, ADAMTS-5, MMP-3, MMP-9 and MMP-13 expression in both SFs and chondrocytes was all repressed by low dose of 2ccPA. Even after cells had been stimulated by cytokines, $10 \mu \mathrm{M}$ 2ccPA repressed expression of cartilage degenerating enzymes in SFs or chondrocytes. Expression of MMP-13 were repressed more in chondrocytes by 2ccPA. ELISA results also confirmed the inhibitory effect of 2ccPA on MMP-13 production in RA SFs $(n=5)$ by $52 \%$ or in RA chondrocytes $(n=3)$ by $43 \%$. In OA, MMP-13 production was reduced by $31 \%$ in OA SFs $(n=6)$ and $34 \%$ in OA chondrocytes $(n=4)$. However, not significant reduction of MMP-3 in both SFs or chondrocytes. MMP-9 expression by ELISA was under the detectable limit. 